Eastern Economic Journal, 2013, 39, (424)

(C) 2013 EEA 0094-5056/13

www.palgrave-journals.com/eej/

Corrigendum

\title{
Single Mothers' Time Preference, Smoking, and Enriching Childcare: Evidence from Time Diaries
}

Sabrina Wulff Pabilonia and Younghwan Song

Eastern Economic Journal (2013) 39, 424. doi:10.1057/eej.2013.27

Correction to: Eastern Economic Journal (2013), 39(2), 227-255.

doi:10.1057/eej.2013.7

Equations 4 and 5 published on Page 230 were incorrect and appeared as follows:

$$
\frac{\partial T_{c}}{\partial r}=\frac{\left(\frac{1}{1+r}\right)^{2} C^{\prime}}{\frac{1}{1+r} C^{\prime \prime}+\eta^{\prime \prime}}<0
$$

and the effect of the discount rate on childfree leisure is positive:

$$
\frac{\partial T_{L}}{\partial r}=\frac{\left(\frac{1}{1+r}\right)^{2} C^{\prime}}{-\frac{1}{1+r} C^{\prime \prime}-\eta^{\prime \prime}}>0
$$

The above equations should have appeared as follows:

$$
\frac{\partial T_{c}}{\partial r}=\frac{\alpha^{\prime \prime} w^{2}\left(\frac{1}{1+r}\right)^{2} C^{\prime}+\eta^{\prime \prime}\left(\frac{1}{1+r}\right)^{2} C^{\prime}}{\eta^{\prime \prime} \alpha^{\prime \prime} w^{2}+\left(\frac{1}{1+r}\right) C^{\prime \prime} \alpha^{\prime \prime} w^{2}+\left(\frac{1}{1+r}\right) C^{\prime \prime} \eta^{\prime \prime}}<0
$$

and the effect of the discount rate on childfree leisure is positive:

$$
\frac{\partial T_{L}}{\partial r}=\frac{-\alpha^{\prime \prime} w^{2}\left(\frac{1}{1+r}\right)^{2} C^{\prime}}{\eta^{\prime \prime} \alpha^{\prime \prime} w^{2}+\left(\frac{1}{1+r}\right) C^{\prime \prime} \alpha^{\prime \prime} w^{2}+\left(\frac{1}{1+r}\right) C^{\prime \prime} \eta^{\prime \prime}}>0
$$

The authors would like to thank Michael Grossman for pointing out this error. 